\title{
Unpacking The Autism Spectrum Disorder Profile of Children With De Novo Disruptive GRIN2B Variants: A Quantitative and Qualitative Analysis
}

Caitlin M. Hudac ( $\square$ chudac@uw.edu )

University of Washington https://orcid.org/0000-0002-0478-440X

Evangeline Kurtz-Nelson

University of Washington

Tara M. Rutter

University of Washington

Jessica L. Peterson

Seattle Children's Autism Center

Kendra Hoekzema

University of Washington

Jennifer Beighley

University of Washington

Morgan E. Kelly

University of Washington

Arianne S. Wallace

University of Washington

Jennifer Gerdts

University of Washington

\section{Evan E. Eichler}

University of Washington

Raphael A. Bernier

University of Washington

Rachel K. Earl

University of Washington

\section{Research}

Keywords: GRIN2B, autism spectrum disorder, genetic etiology, social impairment, restricted and repetitive behaviors

Posted Date: September 18th, 2020

DOI: https://doi.org/10.21203/rs.3.rs-76658/v1

License: (c) (i) This work is licensed under a Creative Commons Attribution 4.0 International License. Read Full License 


\section{Abstract}

Background: Disruptive mutations to GRIN2B are considered a high-confidence genetic factor of autism spectrum disorder (ASD), yet only 25\% of patients with GRIN2B mutations are given a formal ASD diagnosis. Here, we generate a comprehensive understanding of the ASD profile in children with de novo disruptive GRIN2B variants, including social communication deficits and restricted and repetitive behaviors (RRBs).

Methods: The ASD profile was measured in 34 children with disruptive GRIN2B variants, including clinically assessed ASD severity (subset of 16 children) and parental reports of ASD symptomology. GRIN2B ASD profiles were compared to 96 children with a different pathogenic ASDassociated mutation, as well as 55 children with ASD stratified based upon low $(n=14)$ or average $(n=41)$ nonverbal cognition. Following statistical group comparisons, unique GRIN2B ASD features were further characterized using qualitative analysis of clinical observations and case reports.

Results: Both GRIN2B and pathogenic groups exhibited similar proportions of formal ASD diagnoses and clinically assessed ASD severity was similar across comparison groups. Parents endorsed fewer problematic social behaviors and impairing RRBs in certain categories, highlighting relative strengths in socioemotional reciprocity and fewer reported problems with rigidity or restricted interests. Contextualization of behaviors based upon clinical observations indicated a unique GRIN2B profile describing high motivation to approach others, but often inappropriate social responses. Atypical nonverbal communication and sensory seeking were evident.

Limitations: This study is limited by the small sample size for the clinically assessed GRIN2B subset. Second, the ASD comparison group was older than the GRIN2B or Pathogenic groups. Lastly, we focus on ASD symptomology and do not fully characterize other psychiatric, behavioral, or medical comorbidities or link the ASD profile to the specific function of GRIN2B variants (e.g., loss or gain of function), which may be a critical next step for developing targeted interventions.

Conclusions: We identify a unique ASD profile for children with disruptive GRIN2B variants involving strengths in social motivation/approach and reduced impairing RRBs. Continued work phenotyping GRIN2B should incorporate nuanced examination of these strengths in the context of the challenges faced by individuals with disruptive GRIN2B variants.

\section{Background}

Autism spectrum disorder is a heterogeneous neurodevelopmental disorder (NDD) characterized by social communication impairments, as well as restrictive and repetitive interfering behaviors [1]. Ongoing efforts over the past decade have uncovered critical genetic factors linked to ASD and related neurodevelopmental disorders (e.g., intellectual disability, ID)[2,3], which may elucidate the biological mechanisms of ASD as a crucial step for developing treatments. Single de novo likely gene-disrupting (LGD) mutations and rare copy number variations account for approximately $30 \%$ of simplex ASD cases [4-6], yet the unique constellation of ASD symptomology associated with specific events is limited by the rarity of cases and a lack of in-depth phenotype information.

One such ASD-associated risk gene involves recurrent disruptive mutations in GRIN2B that are associated with ASD and ID [7-12], as well as other childhood-onset neurodevelopmental disorders [13-15]. The GRIN2B gene, located on the short arm of chromosome 12, encodes the GluN2B subunit of the N-Methyl-d-aspartate (NMDA) receptors (NMDARs) widely expressed in the central nervous system. NMDA receptors mediate excitatory neurotransmission and appear to play a crucial role in brain development, learning, and synaptic plasticity $[7,16]$. GluN2B expression is highest during prenatal and postnatal periods, and begins to decline during the postnatal period and become more restricted to the forebrain [17]. Functional disruption of GluN2B subunit and NDMA signaling is theorized to result in disruptions in neuronal connections, synaptic plasticity, and excitatory transmission [7]. Healthy populations demonstrate significantly less variation in the GRIN2B gene than clinical groups, suggesting GRIN2B is likely instrumental to normal neurological functioning and development [12,18].

Despite being implicated as a strong candidate ASD risk gene [14,19], one study suggested only 25\% of patients (23 of 91 patients with phenotype data) present with an ASD phenotype [11]. For instance, although significant social problems are noted on standardized measures, qualitative reports characterize children with GRIN2B mutations as friendly and socially boundless, with a tendency to approach others in a trusting manner [15]. The current investigation aims to delve into a closer inspection of ASD symptomology within a large sample of children with de novo disruptive GRIN2B mutations to better understand GRIN2B as a possible etiology of ASD. To best understand the unique features of this group, the current study will be the first to compare quantitatively assessed clinical and behavioral characteristics in a sample of individuals with de novoGRIN2B variants to patients with disruptive mutations to other high confidence NDD risk genes [20-24], as well as a large comparison group of individuals with ASD but without identified likely gene disrupting (LGD) mutations (i.e., Idiopathic ASD). Importantly, the latter comparison group of children without an LGD event will be stratified by cognition to better disentangle the impact of intellectual disability and cognitive impairments on ASD symptomology. Subsequent to traditional group statistical analyses, we utilize a qualitative approach to contextualize the results via the examination of clinical observations and case reports. These efforts to distinguish a unique 
phenotype that differentiates the GRIN2B presentation from that of ASD (with and without ID) and other genetic ASD subtypes is important for guiding future research and clinical endeavors including identification and treatment with this population.

\section{Materials And Methods}

\section{Participants.}

Recruitment sources included genetic-first studies at the University of Washington (TIGER and ZEBRA), and an additional 19 participants with disruptive GRIN2B mutations were included from the Simons Variation in Individuals Project (SVIP, now known as Simons Searchlight)[25]. Participants were ascertained in two ways based upon group: (1) the presence of a disruptive mutation that is associated with ASD and related neurodevelopmental disorders (GRIN2B target group and Pathogenic comparison group); or (2) the presence of a known ASD diagnosis (ASD comparison group). Main analyses involve a total of 34 individual children with de novo GRIN2B mutations who were compared to three comparison groups: (1) Pathogenic group consisting of 96 age-, sex-, and cognitive-matched children with a different pathogenic, ASDassociated mutation, regardless of whether the child had an ASD diagnosis. Children with ASD and no known ASD-associated disruptive mutation were split into (2) ASD No Event Low IQ ( $\leq 70$ NVIQ, $n=14)$. matched on sex and nonverbal cognition, and (3) ASD No Event Average IQ (85-115 NVIQ, $n=41)$, matched on sex. An extended discussion of developmental considerations due to the lack of an ASD comparison group matched on chronological age is included below as a limitation of the current study.

See Table 1 for full participant characterization and group differences. Comprehensive characterization was conducted with participants by research clinicians either on-site at the University of Washington or via home visit at the participant's home ( $n=13$ for GRIN2B, $n=99$ for Pathogenic). Each ASD No Event participant completed a visit at the University of Washington. Children participating on-site or via home visit completed a cognitive and adaptive testing and received a diagnosis of ID or global developmental delay (GDD) by research clinicians using the DSM-5 [26]. Diagnoses of GDD were given to participants under age 5 years who demonstrated significant impairment in intellectual and adaptive functioning. Additional measures were collected via remote participation (i.e., online surveys and phone calls) for the other remaining participants. All data collection and research procedures were approved by the University of Washington ethical review board, and written and informed consent and/or assent were obtained from all participating individuals capable of consent or assent.

\section{Genetic characterization.}

For GRIN2B and Pathogenic groups, presence of a disruptive variant was confirmed through review of the clinical genetic testing lab report or through targeted or exome sequencing conducted as part of the referring study [24,25]. See SI Table 1 for full genetic characterization. The main analyses are restricted to individuals with single gene mutations deemed to be pathogenic (i.e., mutation to GRIN2B or other ASD-risk associated gene). An additional two participants with a GRIN2B mutation deemed a variant of unknown significance completed a portion of remote research procedures and supplemental analyses with the full GRIN2B sample can be found in SI Table 2.

\section{Measuring the ASD profile:}

\section{Clinically-assessed ASD severity and ASD symptomology.}

Gold standard ASD diagnostic instruments were administered by research reliable clinicians, including the Autism Diagnostic Observation Schedule-Second Edition (ADOS-2)[27] to directly assess ASD symptoms and the Autism Diagnostic Interview -Revised (ADI-R) [28] to assess developmental history and parent report of ASD symptoms. Diagnoses reported by remote parents without confirmation are described as suspected, and diagnoses confirmed by our research team using the DSM-5 [26] are noted as clinically confirmed. Data was analyzed in three ways. First, calibrated severity scores (CSS) were utilized as an ASD severity metric for comparison across ADOS-2 module [29]. Second, to compliment this quantitative assessment of ASD severity, we utilized the ADI-R to characterize ASD symptomology by selecting relevant items to address specific a priori subdomains (SI Table 3 ) that best follow the DSM-V diagnosis criteria and are appropriate for individuals with intellectual disability and/or language impairment (e.g., ID or GDD populations). We examined scores related to both historical and current behaviors to better understand early problems and characterize the current ASD profile. We converted raw scores to the following categorical values: No impairment (scores of 0), Unclear impairment (scores of 1), and Clear impairment (scores of 2 or 3). ADI-R scores of 7, 8, or 9 were considered missing data. Lastly, following quantitative results, we evaluated clinical case reports, clinician notes, and ADOS-2 item scores to qualitatively describe ASD symptoms associated with GRIN2B (described in more depth below).

\section{Parent-reported social ASD symptoms.}

Three metrics were used as primary outcomes. First, parents completed the Social Responsiveness Scale Second edition (SRS-2) [30]. The SRS2 has five subscales assessing various types of social impairment, including social awareness (i.e., the ability to pick up on social cues), social cognition (i.e., the ability to interpret social cues), social communication (i.e., social communication skills including expressive social communication), social motivation (i.e., overall motivation to engage in social-interpersonal behavior), and autistic mannerisms (i.e., stereotyped 
behaviors and/or highly restricted interests). Second, parents completed the Social Communication Questionnaire [31], which serves as a widespread screening measure for ASD in youth [32,33]. Third, parents completed the Vineland Adaptive Behavior Scales, Second Edition (VABSII) [34], which was used to assess adaptive functioning skills in the areas of communication, daily living skills and socialization. For the purposes of this paper and aligned with prior work [35,36], we focus on the socialization subdomain as it pertains to social ASD symptoms. Additional measures, including the Theory of Mind inventory (ToMI) [37,38] and Empathy Quotient (EQ) [39], were collected but had insufficient GRIN2B participants $(n=6)$ for valid conclusions[1]. Analyses for these measures can be found in SI Table 4.

\section{Parent-reported restricted and repetitive behaviors (RRB) ASD symptoms.}

Parents of children in the GRIN2B or Pathogenic groups completed the Repetitive Behavior Scale-Revised (RBS-R) [40], which included a total tscore, as well as t-scores for subdomains of sensorimotor behaviors, restricted interests, self-injurious behaviors, compulsive behaviors, and need for sameness. RBS-R data were unavailable for children with idiopathic ASD.

\section{Quantitative analytic plan.}

A series of linear mixed-effects models were computed in R version 3.6.1 using the Ime4() package [41] to determine main effects of group, with a focus on GRIN2B group differences relative to Pathogenic, ASD No Event Low IQ, and ASD No Event Average IQ. Pairwise comparisons were corrected using Bonferroni correction. Analyses on ADI-R items involved Chi-square tests between qualitative level of impairment (None, Unclear, Clear) and group.

\section{Qualitative analytic plan.}

Lastly, we analyzed clinical observations to qualitatively describe ASD symptoms associated with GRIN2B. Qualitative descriptions of 13 participants with disruptive GRIN2B mutations who completed comprehensive in-person evaluations were examined and coded by a licensed psychologist with expertise in ASD assessment and rare genetic disorders. The clinician reviewed two documents for each participant: (1) clinical case reports and (2) clinician assessment notes. Behaviors of interest among social communication and RRB domains were derived post hoc from ADI-R items, SRS-2 domains, and RBS-R domains where significant differences were identified in the GRIN2B group. Social communication behaviors that were coded included the following: social motivation, quality and frequency of social response, appropriateness of social responses, reciprocal conversation, social disinhibition, showing and directing attention, and pointing to express interest. RRBs included the following: stereotyped/idiosyncratic use of words or phrases, restricted interests, use of objects, or behavior, presence of routines or rituals or difficulties with minor changes, hand and finger or other complex mannerisms, and noted sensory differences. The clinician also reviewed examiner notes for indicators of motor deficits or delay to further characterize quantitative data, particularly to inform conceptualization of RRBs and nonverbal pointing behaviors.

To complement clinical observations, ADOS-2 item-level scores across modules 1, 2, and 3 corresponding to these domains were reviewed and compiled. Again, items reviewed were selected based on the results of quantitative analyses. In the Social Affect category, items measuring the following behaviors were reviewed: pointing, gesture use, amount of social overtures toward examiner or caregiver, quality of social response, quality of rapport with examiner, and amount of reciprocal social communication. In the Restricted and Repetitive Behavior category, items measuring unusual sensory interest, complex body mannerisms, and repetitive interests or stereotyped behavior were reviewed. Participants were then stratified by presence of ASD diagnosis to determine shared and distinct characteristics.

\section{Footnote:}

[1]A priori power analyses using G*Power [74] indicated a minimum of 12 participants per group to achieve $90 \%$ power for large effects ( $a=.05)$.

\section{Results}

\section{Clinically-assessed ASD severity and ASD symptomology.}

As evident in Table 1, from individuals with available data, $75 \%$ of participants with disruptive GRIN2B and $79 \%$ of participants with other pathogenic mutations had a clinician-confirmed or parent-reported diagnosis of ASD. Standardized ADOS-2 CSS scores indicated similar ASD severity for all groups, $F(3,53)=0.90, p=0.45$, including social affect and RRB subdomains, $p$ 's $>0.27$, suggesting that severity of clinicianobserved ASD symptoms is comparable. See Table 2 for descriptive statistics of ADOS-2.

As illustrated within Figure 1 (full proportions available in SI Table 3), the proportion of GRIN2B parents endorsing clear, problematic social behaviors and impairing RRBs is low on several items relative to the comparison groups on the ADI-R, highlighting several potential strengths. For instance, more than half of the GRIN2B children exhibited positive socioemotional reciprocity (e.g., social smiling, shared enjoyment with others) and only a few parents reported problems with routines or restricted interests. Parents did note problems with current friendships (item $65,83.3 \%$ ), though only one of four children (older than 10 years of age for this historical item) were endorsed as having a history of problems 
with friendships between age 10-15 years. Other areas of concern included showing and directing attention (item $52,50.5 \%$ with current and $75 \%$ with abnormal behaviors between $4-5$ years of age) and social disinhibition (item $66,87.5 \%$ with current and $81.3 \%$ with abnormal behaviors between 4-5 years of age).

Statistical results relevant to GRIN2B are reported in Table 3 (see SI Table 3 for full results). GRIN2B and Pathogenic groups exhibited elevated patterns on several items relative to ASD comparison groups, including current problems showing and directing attention (Item 52) and problems pointing to express interest at age 4-5 years (item 42; GRIN2B group also elevated on current behaviors). However, the genetic groups exhibited fewer problems involving rituals and routines (current problems, item 74; lifetime problems, items 70, 74) and restricted or circumscribed interests (lifetime problems, item 68). Two historical items indicated a unique profile for the GRIN2B group. First, only 2 of 16 GRIN2B parents reported problems with appropriateness of social responses at age 4-5 years (item 59) relative to the Pathogenic and ASD Low NVIQ groups. Second, a similarly low proportion of GRIN2B parents (5 out of 16) reported lifetime problems with abnormal, idiosyncratic, or negative responses to sensory stimuli (item 73), relative to both ASD comparison groups.

\section{Parent-reported symptoms.}

Full descriptive statistics are provided in Table 2.

Social responsiveness and social skills as measured via the SRS-2 total score indicated an overall group effect that was clarified by significant effects within subdomains (see Figure 2). Unsurprisingly, given the IQ stratification across groups, the ASD Average group exhibited fewer social cognitive and social communication problems than the genetic groups including the GRIN2B group. Most notably, the GRIN2B group exhibited fewer social motivation problems relative to both ASD No Event groups, $t(64)$ 's $<2.93, p^{\prime} s<.029$, (and numerically relative to the Pathogenic group, $t(624)=2.35, p=.13)$.

Based upon the SCQ as an ASD screener, all groups indicated elevated risk (group means > 11) with a significant group effect but no significant pairwise comparisons after Bonferroni-correction, $p$ 's $>.067$. Of note, although the majority of the GRIN2B group scored within the high ASD risk range, $25 \%$ of the GRIN2B group scored within the low ASD risk range (scores $<=10$ ) relative to $<9 \%$ of the comparison groups, indicating a possible GRIN2B subgroup stratified by ASD risk.

The VABS-2 indicated poor socialization adaptive abilities for GRIN2B, Pathogenic, and ASD Low groups relative to the ASD Average group, $p<$ .0001, which may be tied to the IQ stratification.

Lastly, the ASD Low group exhibited the most prominent repetitive behaviors measured via the RBS-R, particularly related to compulsive behaviors, $p<.0001$. However, the GRIN2B group also exhibited fewer sameness behaviors (e.g., insisting that routines and environment stay the same) relative to ASD Average and ASD Low comparison groups.

\section{Contextualization based upon clinical case reports.}

Evaluation of qualitative data revealed similarities within social communication, RRB, and motor domains across $G R I N 2 B$ participants. Qualitative examiner notes and case reports were available for 13 GRIN2B participants who completed comprehensive evaluations. One participant's ADOS-2 was not scored due to significant validity concerns associated with severe motor and cognitive impairment, so ADOS-2 item scores were available for 12 participants. The majority of participants were described as motivated to socially approach others $(n=11 / 13$; $84 \%)$; however, social approach was often characterized as inappropriate or awkward. In addition, social response was commonly described as limited ( $n=9 / 13,69 \%)$, with noted difficulty sustaining reciprocal conversation among verbal participants $(n=7 / 8 ; 87 \%)$. During examination with the ADOS-2, half of GRIN2B participants made frequent social overtures toward the examiner (item score of $0, n=6 / 12 ; 50 \%$ ); slightly less than half of participants who completed a Module 1 or 2 made frequent social overtures toward their caregiver ( $n=4 / 9 ; 44 \%$ ). However, very few participants engaged in appropriate and effective social overtures (item score of $0, n=2 / 12 ; 17 \%$ ) or responses (item score of $0, n=1 / 12 ; 8 \%$ ).

Participants were also described in clinician notes as having limited or absent pointing to show interest ( $n=9 / 1369 \%$ ). During the ADOS-2, only one proband who completed Module 1 or 2 pointed with an index finger and coordinated gaze to reference distal objects during at least two activities (item score of $0, n=1 / 9 ; 11 \%$ ). However, significant motor difficulties were present across $100 \%$ of GRIN2B participants as reported by clinicians and supported by parental report[1]; these delays may have impacted participants' ability to point with a single index finger. The severity of reported motor delays was highly variable (e.g., ranged from "use of wheelchair" to "difficulty changing speed and direction of walking") and not ascertained systematically, and thus we can only make limited conclusions about the degree to which this finding may be unique to the GRIN2B phenotype.

Within the RRB domain, high rates of restrictive interests and/or repetitive behaviors were present $(n=11 / 13 ; 84 \%)$. Fine-grained examination of clinician notes indicated a majority of GRIN2B participants had a noted sensory difference $(n=10 / 13 ; 76 \%)$, with over one-third $(n=5 ; 38 \%)$ presenting with a hypersensitivity to sound, likely indicating variable presentation of sensory sensitivity. Additionally, frequent mouthing and 
licking of objects was noted in $30 \%$ of participants $(n=4 / 13 ; 31 \%)$. Atypical hand and arm movements (e.g., hand mannerisms, hand flapping, finger posturing) were also highly prevalent within the sample $(n=9 / 1369 \%)$. The majority of participants demonstrated clear complex mannerisms during the ADOS-2 (item score of 2 or $3, n=7 / 12 ; 58 \%$ ). Less than half of participants demonstrated clear repetitive or stereotyped interests or behaviors (item score of 2 or $3, n=5 / 12 ; 42 \%$ ). Clear unusual sensory interests were not observed frequently during the ADOS-2 (item score of 2 or $3, n=3 / 12 ; 25 \%$ ); however, mouthing of objects would likely not be coded in this section per ADOS-2 scoring guidelines.

GRIN2B participants were then stratified by ASD diagnosis to determine qualitative ASD profile differences for children who did not receive an ASD diagnosis by the clinical research team. Among the non-ASD GRIN2B group $(n=5)$, two participants presented with profound cognitive and motor impairments that complicated diagnostic confirmation and impacted ADOS-2 assessment validity. The remaining three participants were noted by clinicians to have social communication skills commensurate with their developmental level. Of these three participants, all engaged in frequent social overtures toward the examiner (item score of $0, n=3 / 3,100 \%$ ) and their caregiver (item score of $0, n=3 / 3,100 \%$ ) during the ADOS-2. Clear impairments in quality of social overture and response were also not observed for these participants (item score of 2 or $3, n=0 / 3$; $0 \%$ ). Within the non-ASD GRIN2B group, all participants were characterized as motivated to approach others $(n=5 / 5 ; 100 \%)$, with over half of the participants noted as demonstrating appropriate social response $(n=3 / 5 ; 60 \%)$. Participants were also described as having limited or absent pointing to show interest $(n=4 / 5 ; 80 \%)$, which was consistent with ADOS-2 scores (item score of $0,0 / 3 ; 0 \%$ ). A majority $(n=3 / 5 ; 60 \%)$ demonstrated restrictive interests and/or repetitive behaviors. Again, fine-grained examination of clinician notes indicated a majority of non-ASD GRIN2B participants had a noted sensory difference $(n=4 / 5,80 \%)$, with half noted to have a hypersensitivity to sound ( $n=2 / 4$; $50 \%)$, and half reported to engage in frequent mouthing/licking of objects $(n=2 / 4,50 \%)$. All three probands whose social communication skills were reportedly commensurate with their developmental level displayed at least one clear restricted or repetitive behavior during the ADOS-2 (item score of 2 or 3); two demonstrated complex mannerisms, one demonstrated an unusual sensory interest, and one demonstrated a stereotyped interest or behavior.

\section{Footnote:}

[1] Based upon parents who completed the Developmental Coordination Disorder (DCD) Questionnaire [75], all GRIN2B parents ( $n=17 / 17 ; 100 \%)$ indicated or suspected DCD.

\section{Discussion}

In this study, we generated a comprehensive description of the ASD profile in children with de novo disruptive GRIN2B variants to elucidate unique features relative to other LGD and ASD comparison groups. Our findings indicate a distinct GRIN2B ASD phenotype that displayed fewer problematic social behaviors and impairing RRBs. Specifically, we note intact socioemotional reciprocity and fewer reported challenges with the rigidity of routines or restricted interests, which were supported and contextualized by clinical observations. Here, we discuss the constellation of features in more depth and describe implications for clinical outcomes and treatment.

\section{Strengths in socioemotional reciprocity.}

Among children with de novo GRIN2B variants, socioemotional reciprocity appears to be a unique strength of the phenotype relative to LGD and idiopathic ASD groups. Social smiling and shared enjoyment with others were also reported in over half the GRIN2B group. Historical lifetime prevalence of reciprocal friendships also confirms an intact social motivation profile. Roughly half of the GRIN2B sample demonstrated frequent social overtures towards examiners or caregivers as indicated by ADOS-2 item scores. Additionally, parent report on the SRS-2 indicated that GRIN2B participants exhibited less social motivation difficulties compared to the idiopathic ASD group. This is in contrast to SRS-2 profiles among several known ASD-associated mutation groups; for example, individuals with CHD 8 and FMR1 mutations are characterized by notably low social motivation [42,43]. However, items within the social motivation SRS-2 subscale are relatively diverse, including approach/avoidance of social situations (e.g., "Would rather be alone than with others"), aspects of self-confidence, and symptoms of social anxiety (e.g., seems much more fidgety in social situations than when alone). Given previously identified associations between SRS scores and genetic risk [44], it may be helpful to engage in a more fine-grained item-level analyses to better specify areas of social strengths in GRIN2B children that can be captured by parental report.

Extending from clinical observations noted within the case reports and clinical descriptions, GRIN2B participants were frequently characterized as demonstrating high social motivation and approach, consistent with quantitative data across both gold-standard clinical assessments and parent-report. Overall, the social exuberance observed in the current sample is consistent with previous characterizations describing individuals with de novoGRIN2B mutations as "trusting" and "socially boundless"[15]. Interestingly, qualitative notes stratified by ASD diagnosis revealed similar profiles of social motivation and approach, such that regardless of whether the child met strict ASD diagnostic criteria, socioemotional reciprocity was noted as a strength. Continued phenotyping work is encouraged to delineate and identify how social strengths may be unique in GRIN2B populations and can be leveraged to bolster social skills and communication interventions. 
Despite social strengths in motivation and approach, GRIN2B social profiles also demonstrate consistently inappropriate or limited social response. ADI-R items indicated elevated problems as a young child with appropriateness of social responses (item 59) compared to the pathogenic and ASD Low NVIQ groups. Within qualitative data examined, social response was commonly described as limited, including clinical descriptions such as "socially naïve, unsure what to say and do", "does not respond to attempts by others to engage", and "inappropriate facial expressions (hysterical laughter)". Participants with verbal skills also demonstrated noted difficulty sustaining reciprocal conversation. Clinical characterizations of this difficulty included a limited ability to sustain conversation outside of the participant's own interests and requiring repeated probes to report on events.

As impairments in social interactions are a key diagnostic feature of ASD, it is critical to characterize differences across social motivation and social response among etiologically distinct subgroups to inform precision medicine. One theory is that early alterations to social motivation and reward appear to alter underlying mechanisms that ultimately disrupt social processing in ASD [45], supported by work in neuroimaging [46-48]. It may be possible that social motivation serves as a protective factor for children with de novo GRIN2B mutations, whereas inappropriate social responses may reflect underlying social misjudgment [49]. Prior work in a large ASD sample indicated that despite evidence that high social motivation can facilitate social skills, social dysregulation (e.g., internalization, aggressive behaviors, irritability) may interfere with positive outcomes [50]. Because social response appears to be a limitation to the GRIN2B social profile, social skills interventions could target these specific challenges (e.g., reciprocal conversation skills and appropriate response to the approach of others). Additionally, given current efforts to determine how pharmacological intervention may be able to rescue social function in animal models [51], continuing to finetune what social functions should be targeted in GRIN2B (and other genetic etiologies of ASD) will be critical.

Perhaps related to these inappropriate social responses, children with de novoGRIN2B variants were much more likely to receive an ASD diagnosis than population estimates of $\sim 1.6 \%$ [52]. In our sample, 8/13 (62\%) of those receiving an in-person research assessment received an ASD diagnosis by a licensed clinical psychologist using DSM-5 criteria [26]. The intact social interest and social disinhibition widely observed in GRIN2B is similar to the "active-but-odd" social interaction subtype originally proposed by Wing and Gould in 1979 [53]. This style of interaction is characterized by active seeking of contact and interactions, although social initiations and responses are generally unusual and/or inappropriate (i.e., personal space boundary violations, asking personal questions, talking at length about a circumscribed or unusual interest). The active-but-odd interaction style in ASD has been associated with higher cognitive ability and adaptive skills, decreased autism severity, motor deficits, and attention deficit and hyperactivity symptoms [54-56]. Our results further expand previous characterizations of GRIN2B social interactions, such that approach appears intact but response is limited, which may potentially be linked to specific molecular mechanisms $[11,14,15]$.

\section{Disentangling the role of cognition in ASD symptomology.}

All GRIN2B participants who completed cognitive and adaptive testing met criteria for ID or GDD, providing additional evidence for the connection between ID and disruptive mutations to this gene $[8,11,14,15,57]$. Diagnosis of ASD in the context of ID is challenging, particularly among individuals with severe to profound ID or co-occurring motor, vision, or hearing impairments [58]. Standardized assessments of ASD symptoms (e.g., the ADOS-2, ADI-R, and SRS-2) have reduced specificity and validity for individuals with severe cognitive, motor, or sensory impairment, so clinical judgment is needed when using these instruments to inform diagnosis [58-60]. Social communication deficits as compared to same-age peers are characteristic of ID; as such, it may be difficult to determine whether observed social communication deficits are inconsistent with developmental level as opposed to chronological age [26,60,61].

Additionally, there is considerable overlap in the restricted and repetitive behaviors observed in individuals with ASD and comorbid ID and individuals with ID only. Specifically, similar rates of repetitive actions on objects, need for sameness, repetitive motor mannerisms, preoccupation with parts of objects, and nonfunctional routines and rituals have been found in both populations [62-66]. DSM-5 criteria that appear to best distinguish between ASD with ID and ID only include poor nonverbal communication (e.g., coordinated eye contact), reduced showing and sharing of interests, limited social emotional reciprocity, and the presence of restricted interests being related to ASD [63,64]. Differentiating the role of cognitive impairment in the presentation of ASD symptoms in individuals with GRIN2B is complicated by the many overlapping clinical features in both neurodevelopmental conditions.

Overall, individuals with ID-associated genetic disorders often present with a complex and idiosyncratic profile of cognitive, language, motor, medical, psychiatric, and behavioral impairments, which may contribute to over diagnosis or inconsistent diagnosis of ASD in genetic disorders [58,67-69]. Future research on ASD profiles in GRIN2B and other rare ASD-associated genetic disorders should continue to incorporate novel measures that are aligned with developmental level and validated for individuals with severe to profound ID [58-61,70].

\section{Limitations of the current study.}


The findings of this study should be considered in the context of study limitations. First, while this study provides an in-depth look at the ASD profile of the largest GRIN2B cohort to date, the sample size, particularly those with clinically-assessed ASD severity, remains small, which limited quantitative analyses and the extent to which findings can be applied broadly to the GRIN2B population. Within the current study, GRIN2B cases were identified from two different sources, each of which differed in the amount of clinical phenotyping that was conducted and availability of complete data across cases. Due to the small sample size, we adopted the traditional (albeit potentially problematic [71]) statistical significance threshold of $p<0.05$ that may have contributed spurious findings. Given that the goal of this study was to provide an initial characterization of the comprehensive GRIN2B ASD profile and was not assessing efficacy of a clinical trial, we argue that this threshold was sufficient for our purposes. However, continued phenotyping efforts are needed to increase size of future cohorts with particular attention paid to harmonizing quantitative assessment tools for consistent characterization of patients.

A limitation of comparisons between GRIN2B, Pathogenic, and ASD comparison groups should also be noted. While GRIN2B and Pathogenic groups were matched on sex, age, and cognitive ability, an age-matched "idiopathic" ASD comparison group was not available for analyses. The ASD comparison group was significantly older than the GRIN2B and Pathogenic groups, which may impact symptom presentation comparisons across groups. Future studies would benefit from an age-matched comparison group to further understand differences in $G R I N 2 B$ and idiopathic ASD profiles.

The current study focused on clarifying and informing socioemotional and RRB profiles for GRIN2B. However, as with many high-confidence neurodevelopmental disorder risk genes, GRIN2B is also characterized by significant medical and psychiatric comorbidities, which were not the focus of this study. Medical conditions including hypotonia, gastrointestinal disturbances, sleep difficulties, and seizures have been noted in GRIN2B at rates consistent with other neurodevelopmental disorder risk genes. Additionally, attention difficulties and hyperactivity are frequently reported in those with GRIN2B variants. These medical and psychiatric comorbidities are likely to impact development and behavioral presentation and should be considered in future analyses to clarify the communication, social, and behavioral profiles of patients with $G R I N 2 B$ mutations.

Lastly, the current study did not evaluate phenotypic presentation of GRIN2B variants in tandem with a functional characterization of the genetic variants. Recent publications report a range of functional consequences of disruptive mutations in GRIN2B and suggest a possible link between gain of function mutations and epilepsy, although significantly more research is needed in this area [8,11]. Additional multidisciplinary collaborations that connect functional mechanisms of GRIN2B to clinical presentation is essential to understanding the role of GRIN2B in NDD risk and identifying points of pharmacological and behavioral intervention.

\section{Conclusion}

Our study aimed to elucidate ASD symptomology among children with de novo disruptive GRIN2B variants. Building upon other behavioral phenotyping efforts [15], we examined the profile of social communication impairments and RRBs in GRIN2B as compared to other LGD and idiopathic ASD groups. Our multi-informant approach provides a robust contextualization of ASD profiles and is a strength of this investigation. Both quantitative and qualitative data converged on a clear profile of social communication skills and RRBs unique to GRIN2B. While overall ASD severity was similar across groups, GRIN2B individuals exhibited intact social motivation and approach, limited social response, reduced pointing, noted sensory differences, atypical hand and arm mannerisms, and fewer restricted interests and rituals. Employing a larger sample, our findings confirm prior observations of "friendly but boundless social behavior" [15] and extend phenotype characterization to detail differences across nonverbal communication and an RRB profile characterized by repetitive motor behaviors. This nuanced examination of the phenotype has the potential to inform precision medicine, as individuals with disruptive GRIN2B variants appear to have similar strengths and challenges that may guide intervention recommendations and development of new treatments specific for GRIN2B. For instance, healthcare providers serving GRIN2B patients may find utility in targeting specific social communication limitations, such as pointing to share interest and appropriate social response. In the RRB domain, behavioral interventions to prevent repetitive motor behaviors from progressing to self-injurious behaviors may be warranted [72,73]. Our strong convergence across data sources provides a characterization of an ASD profile unique to GRIN2B, and further phenotyping of GRIN2B and other de novo disruptive variants should incorporate this multi-informant, data-driven approach to examine biologically based ASD profiles to inform precision medicine.

\section{Abbreviations}

ASD, Autism spectrum disorder; ID Intellectual disability; RRBs, restricted and repetitive behaviors

\section{Declarations}

\section{Ethics approval and consent to participate}


Written consent was obtained from participant's parent or legal guardian. Informed assent was obtained from all participating individuals capable of consent or assent. All procedures were approved by the University of Washington Institutional Review Board.

\section{Consent for publication}

None of the data in this manuscript requires a consent for publication because it is all de-identified and no image likeness are included.

\section{Availability of data and materials:}

The dataset supporting the conclusions of this article are available upon reasonable request to the lead author, C.M.H.

\section{Competing interests}

E.E.E. is an investigator of the Howard Hughes Medical Institute. None of the other authors have competing interests to report.

\section{Funding}

This research was supported, in part, by the US National Institutes of Health (NIH R01MH101221 to E.E.E.) and the GRIN2B Foundation (Research Grant to C.M.H.).

\section{Author contributions}

The study was conceived and designed by C.M.H., J.P., and R.A.B. Data were collected by C.M.H., E.K-N., T.M.R., J.L.P., J.B., M.K., A.S.W., J.G., R.A.B., and R.K.E. Genetic characterization and interpretation were conducted by K.H. and E.E.E. Quantitative analyses were conducted by C.M.H.., qualitative analyses were conducted by E.K.N. and T.M.R. Results were interpreted by all authors C.M.H., E.K-N.,T.M.R., J.L.P., and R.K.E. wrote the paper with edits from all other authors. All authors approved the final version of this manuscript.

\section{Acknowledgements}

We emphatically thank the families who took part in this study for their time and efforts.

\section{References}

1. Lord C, Brugha TS, Charman T, Cusack J, Dumas G, Frazier T, et al. Autism spectrum disorder. Nat Rev Dis Primers. 2020;6:5.

2. Geschwind DH. Advances in autism. Annu Rev Med. 2009;60:367 380.

3. Grove J, Ripke S, Als TD, Mattheisen M, Walters RK, Won H, et al. Identification of common genetic risk variants for autism spectrum disorder. Nat Genet. 2019;51:431-44.

4. Iossifov I, O’Roak BJ, Sanders SJ, Ronemus M, Krumm N, Levy D, et al. The contribution of de novo coding mutations to autism spectrum disorder. Nature. 2014;515:216 221.

5. Arnett AB, Trinh S, Bernier RA. The state of research on the genetics of autism spectrum disorder: methodological, clinical and conceptual progress. Curr Opin Psychology. 2018;27:1-5.

6. O'Roak BJ, Stessman HA, Boyle EA, Witherspoon KT, Martin B, Lee C, et al. Recurrent de novo mutations implicate novel genes underlying simplex autism risk. Nat Commun. 2014;5:5595.

7. Hu C, Chen W, Myers SJ, Yuan H, Traynelis SF. Human GRIN2B variants in neurodevelopmental disorders. J Pharmacol Sci. 2016;132:11521.

8. Mishra N, Kouzmitcheva E, Orsino A, Minassian B. Chromosome 12p Deletion Spanning the GRIN2B Gene Presenting With a Neurodevelopmental Phenotype: A Case Report and Review of Literature. Child Neurology Open. 2016;3:2329048X16629980.

9. O’Roak B, Vives L, Fu W, Egertson J, Stanaway I, Phelps I, et al. Multiplex Targeted Sequencing Identifies Recurrently Mutated Genes in Autism Spectrum Disorders. Science. 2012;338:1619-22.

10. Pan Y, Chen J, Guo H, Ou J, Peng Y, Liu Q, et al. Association of genetic variants of GRIN2B with autism. Sci Rep-uk. 2015;5:8296.

11. Platzer K, Yuan H, Schütz H, Winschel A, Chen W, Hu C, et al. GRIN2B encephalopathy: novel findings on phenotype, variant clustering, functional consequences and treatment aspects. J Med Genet. 2017;54:460.

12. Yoo HJ, Cho IH, Park M, Yang SY, Kim SA. Family based association of GRIN2A and GRIN2B with Korean autism spectrum disorders. Neurosci Lett. 2012;512:89-93.

13. Dorval KM, Wigg KG, Crosbie J, Tannock R, Kennedy JL, Ickowicz A, et al. Association of the glutamate receptor subunit gene GRIN2B with attention-deficit/hyperactivity disorder. Genes Brain Behav. 2007;6:444-52. 
14. Endele S, Rosenberger G, Geider K, Popp B, Tamer C, Stefanova I, et al. Mutations in GRIN2A and GRIN2B encoding regulatory subunits of NMDA receptors cause variable neurodevelopmental phenotypes. Nat Genet. 2010;42:1021-6.

15. Freunscht I, Popp B, Blank R, Endele S, Moog U, Petri H, et al. Behavioral phenotype in five individuals with de novo mutations within the GRIN2B gene. Behav Brain Funct. 2013;9:20.

16. Tarabeaux J, Kebir O, Gauthier J, Hamdan F, Xiong L, Piton A, et al. Rare mutations in N-methyl-D-aspartate glutamate receptors in autism spectrum disorders and schizophrenia. Transl Psychiat. 2011;1:e55-e55.

17. Loftis JM, Janowsky A. The N-methyl-d-aspartate receptor subunit NR2B: localization, functional properties, regulation, and clinical implications. Pharmacol Therapeut. 2003;97:55-85.

18. Petrovski S, Wang Q, Heinzen EL, Allen AS, Goldstein DB. Genic intolerance to functional variation and the interpretation of personal genomes. Plos Genet. 2013;9:e1003709.

19. O’Roak BJ, Deriziotis P, Lee C, Vives L, Schwartz JJ, Girirajan S, et al. Exome sequencing in sporadic autism spectrum disorders identifies severe de novo mutations. Nat Genet. 2011;43:585 589.

20. Bernier R, Golzio C, Xiong B, Stessman HA, Coe BP, Penn O, et al. Disruptive CHD8 mutations define a subtype of autism early in development. Cell. 2014;158:263 276.

21. lossifov I, Levy D, Allen J, Ye K, Ronemus M, Lee Y, et al. Low load for disruptive mutations in autism genes and their biased transmission. Proc National Acad Sci. 2015;112:201516376 12.

22. Cotney J, Muhle RA, Sanders SJ, Liu L, Willsey AJ, Niu W, et al. The autism-associated chromatin modifier CHD8 regulates other autism risk genes during human neurodevelopment. Nat Commun. 2015;6:1 11.

23. Sanders SJ, Murtha MT, Gupta AR, Murdoch JD, Raubeson MJ, Willsey AJ, et al. De novo mutations revealed by whole-exome sequencing are strongly associated with autism. Nature. 2012;485:237 241.

24. Stessman HAF, Xiong B, Coe BP, Wang T, Hoekzema K, Fenckova M, et al. Targeted sequencing identifies 91 neurodevelopmental- disorder risk genes with autism and developmental-disability biases. Nat Genet. 2017;49:1 16.

25. Consortium SV. Simons Variation in Individuals Project (Simons VIP): a genetics-first approach to studying autism spectrum and related neurodevelopmental disorders. Neuron. 2012;73:1063 1067.

26. Association AP. Diagnostic and Statistical Manual of Mental Disorders (DSM-5®). American Psychiatric Pub; 2013.

27. Lord C, Rutter M, Goode S, Heemsbergen J, Jordan H, Mawhood L, et al. Austism diagnostic observation schedule: A standardized observation of communicative and social behavior. J Autism Dev Disord. 1989;19:185 212.

28. Lord C, Rutter M, Couteur AL. Autism Diagnostic Interview-Revised: a revised version of a diagnostic interview for caregivers of individuals with possible pervasive developmental disorders. Journal of Autism and Developmental Disorders. 1994;24:659 685.

29. Gotham K, Pickles A, Lord C. Standardizing ADOS Scores for a Measure of Severity in Autism Spectrum Disorders. J Autism Dev Disord. 2009;39:693-705.

30. Frazier T, Ratliff K, Gruber C, Zhang Y, Law P, Constantino J. Confirmatory factor analytic structure and measurement invariance of quantitative autistic traits measured by the Social Responsiveness Scale-2. Autism. 2014;18:31-44.

31. Rutter M, Bailey A, Lord C. The Social Communication Questionnaire. 2003.

32. Barnard-Brak L, Brewer A, Chesnut S, Richman D, Schaeffer AM. The sensitivity and specificity of the social communication questionnaire for autism spectrum with respect to age. Autism Res Official J Int Soc Autism Res. 2015;9:838-45.

33. CHANDLER S, CHARMAN T, BAIRD G, SIMONOFF E, LOUCAS T, MELDRUM D, et al. Validation of the Social Communication Questionnaire in a Population Cohort of Children With Autism Spectrum Disorders. J Am Acad Child Adolesc Psychiatry. 2007;46:1324-32.

34. Sparrow SS, Balla DA, Cicchetti DV. Vineland II: Vineland adaptive behavior scales. American Guidance Service; 2005.

35. Frost KM, Hong N, Lord C. Correlates of Adaptive Functioning in Minimally Verbal Children With Autism Spectrum Disorder. Am J Intellect Dev Disabil. 2017;122:1-10.

36. Voss C, Schwartz J, Daniels J, Kline A, Haber N, Washington P, et al. Effect of Wearable Digital Intervention for Improving Socialization in Children With Autism Spectrum Disorder: A Randomized Clinical Trial. Jama Pediatr. 2019;173:446.

37. Lerner MD, Hutchins TL, Prelock PA. Brief Report: Preliminary Evaluation of the Theory of Mind Inventory and its Relationship to Measures of Social Skills. J Autism Dev Disord. 2010;41:512 517.

38. Hutchins TL, Prelock PA, Bonazinga L. Psychometric Evaluation of the Theory of Mind Inventory (ToMI): A Study of Typically Developing Children and Children with Autism Spectrum Disorder. J Autism Dev Disord. 2012;42:327-41.

39. LAWRENCE EJ, SHAW P, BAKER D, BARON-COHEN S, DAVID AS. Measuring empathy: reliability and validity of the Empathy Quotient. Psychol Med. 2004;34:911-20. 
40. Lam KSL, Aman MG. The Repetitive Behavior Scale-Revised: independent validation in individuals with autism spectrum disorders. J Autism Dev Disord. 2007;37:855 866.

41. Bates D, Mächler M, Bolker B, Walker S. Fitting Linear Mixed-Effects Models using Ime4. Arxiv. 2014;

42. Beighley JS, Hudac CM, Arnett AB, Peterson J, Gerdts JA, Wallace AS, et al. Clinical and behavioral phenotypes of carriers of mutations in CHD8 or its conserved targets. n.d.;

43. Feinstein C, Singh S. Social Phenotypes in Neurogenetic Syndromes. Child Adol Psych Cl. 2007;16:631-47.

44. Lowe JK, Werling DM, Constantino JN, Cantor RM, Geschwind DH. Social Responsiveness, an Autism Endophenotype: Genomewide Significant Linkage to Two Regions on Chromosome 8. Am J Psychiat. 2015;172:266 275.

45. Chevallier C, Kohls G, Troiani V, Brodkin ES, Schultz RT. The social motivation theory of autism. Trends Cogn Sci. 2012;16:231 239.

46. Larson MJ, South M, Krauskopf E, Clawson A, Crowley MJ. Feedback and reward processing in high-functioning autism. Psychiat Res. 2011;187:198 203.

47. Dichter GS, Richey JA, Rittenberg AM, Sabatino A, Bodfish JW. Reward Circuitry Function in Autism During Face Anticipation and Outcomes. J Autism Dev Disord. 2012;42:147-60.

48. Zeeland AAS-V, Dapretto M, Ghahremani DG, Poldrack RA, Bookheimer SY. Reward processing in autism. Autism Res. $2010 ; 3: 53$ 67.

49. Loveland KA, Pearson DA, Tunali-Kotoski B, Ortegon J, Gibbs MC. Judgments of Social Appropriateness by Children and Adolescents with Autism. J Autism Dev Disord. 2001;31:367-76.

50. Neuhaus E, Webb SJ, Bernier RA. Linking social motivation with social skill: The role of emotion dysregulation in autism spectrum disorder. Dev Psychopathol. 2019;31:931-43.

51. Kim J-W, Park K, Kang RJ, Gonzales ELT, Kim DG, Oh HA, et al. Pharmacological modulation of AMPA receptor rescues social impairments in animal models of autism. Neuropsychopharmacol Official Publ Am Coll Neuropsychopharmacol. 2018;44:314-23.

52. Knopf A. Autism rates increase slightly: CDC. Brown Univ Child Adolesc Behav Lett. 2018;34:4-5.

53. Wing L, Gould J. Severe impairments of social interaction and associated abnormalities in children: Epidemiology and classification. J Autism Dev Disord. 1979;9:11-29.

54. Scheeren AM, Koot HM, Begeer S. Social Interaction Style of Children and Adolescents with High-Functioning Autism Spectrum Disorder. J Autism Dev Disord. 2012;42:2046-55.

55. Castelloe P, Dawson G. Subclassification of children with autism and pervasive developmental disorder: A questionnaire based on Wing's Subgrouping scheme. J Autism Dev Disord. 1993;23:229-41.

56. Ghaziuddin M. Defining the Behavioral Phenotype of Asperger Syndrome. J Autism Dev Disord. 2007;38:138-42.

57. Lemke JR, Hendrickx R, Geider K, Laube B, Schwake M, Harvey RJ, et al. GRIN2B mutations in West syndrome and intellectual disability with focal epilepsy. Ann Neurol. 2014;75:147-54.

58. Thurm A, Farmer C, Salzman E, Lord C, Bishop S. State of the Field: Differentiating Intellectual Disability From Autism Spectrum Disorder. Frontiers Psychiatry. 2019;10:526.

59. Gergoudis K, Weinberg A, Templin J, Farmer C, Durkin A, Weissman J, et al. Psychometric Study of the Social Responsiveness Scale in Phelan-McDermid Syndrome. Autism Res Official J Int Soc Autism Res. 2020;

60. Kidd SA, Berry-Kravis E, Choo TH, Chen C, Esler A, Hoffmann A, et al. Improving the Diagnosis of Autism Spectrum Disorder in Fragile X Syndrome by Adapting the Social Communication Questionnaire and the Social Responsiveness Scale-2. J Autism Dev Disord. $2019 ; 1-20$.

61. Bishop S, Farmer C, Kaat A, Georgiades S, Kanne S, Thurm A. The Need for a Developmentally Based Measure of Social Communication Skills. J Am Acad Child Psy. 2019;58:555-60.

62. Lord C, Pickles A, McLennan J, Rutter M, Bregman J, Folstein S, et al. Diagnosing Autism: Analyses of Data from the Autism Diagnostic Interview. J Autism Dev Disord. 1997;27:501-17.

63. Hartley SL, Sikora DM. Detecting Autism Spectrum Disorder in Children With Intellectual Disability: Which DSM-IV-TR Criteria Are Most Useful? Focus Autism Dev Dis. 2010;25:85-97.

64. Pedersen AL, Pettygrove S, Lu Z, Andrews J, Meaney FJ, Kurzius-Spencer M, et al. DSM Criteria that Best Differentiate Intellectual Disability from Autism Spectrum Disorder. Child Psychiatry Hum Dev. 2017;48:537-45.

65. Vig S, Jedrysek E. Autistic Features in Young Children with Significant Cognitive Impairment: Autism or Mental Retardation? J Autism Dev Disord. 1999;29:235-48.

66. Stone WL, Hogan KL. A structured parent interview for identifying young children with autism. J Autism Dev Disord. 1993;23:639-52.

67. Kurtz-Nelson EC, Beighley JS, Hudac CM, Gerdts J, Wallace AS, Hoekzema K, et al. Co-occurring medical conditions among individuals with ASD-associated disruptive mutations. Child Heal Care. 2020;1-24. 
68. Oberman LM, Kaufmann WE. Autism Spectrum Disorder Versus Autism Spectrum Disorders: Terminology, Concepts, and Clinical Practice. Frontiers Psychiatry. 2020;11:484.

69. Oberman LM, Boccuto L, Cascio L, Sarasua S, Kaufmann WE. Autism spectrum disorder in Phelan-McDermid syndrome: initial characterization and genotype-phenotype correlations. Orphanet J Rare Dis. 2015;10:105.

70. Soorya L, Leon J, Trelles MP, Thurm A. Framework for assessing individuals with rare genetic disorders associated with profound intellectual and multiple disabilities (PIMD): the example of Phelan McDermid Syndrome. Clin Neuropsychologist. 2017;32:1226-55.

71. Wayant C, Scott J, Vassar M. Evaluation of Lowering the P Value Threshold for Statistical Significance From .05 to .005 in Previously Published Randomized Clinical Trials in Major Medical Journals. Jama. 2018;320:1813-5.

72. Fodstad JC, Kirsch A, Faidley M, Bauer N. Demonstration of Parent Training to Address Early Self-Injury in Young Children with Intellectual and Developmental Delays. J Autism Dev Disord. 2018;48:3846-57.

73. Richman DM. Annotation: Early intervention and prevention of self-injurious behaviour exhibited by young children with developmental disabilities: Self-injurious behaviour. J Intell Disabil Res. 2007;52:3-17.

74. Faul F, Erdfelder E, Lang A-G, Buchner A. G*Power 3: A flexible statistical power analysis program for the social, behavioral, and biomedical sciences. Behav Res Methods. 2007;39:175-91.

75. Wilson BN, Crawford SG, Green D, Roberts G, Aylott A, Kaplan BJ. Psychometric properties of the revised Developmental Coordination Disorder Questionnaire. Phys Occup Ther Pedi. 2009;29:182-202.

\section{Tables}

Table 1. Demographic characterization of participants. Group differences for (1) GRIN2B, (2) Pathogenic, (3) ASD Low NVIQ, and (4) ASD Average NVIQ groups are based upon chi-square (qualitative) and one-way ANOVA with Tukey HSD correction (quantitative) analyses.

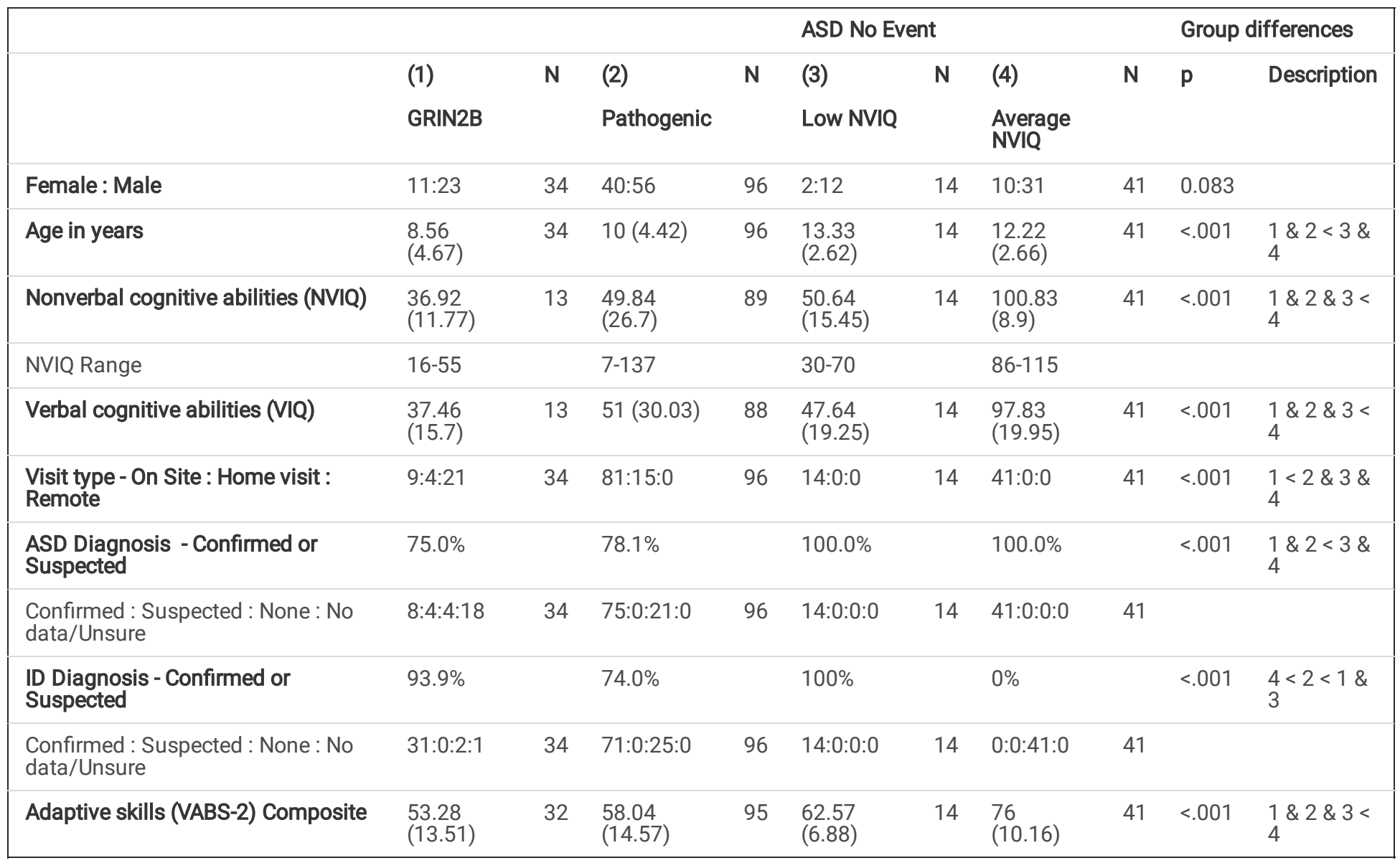

Table 2. ADOS-2 characterization and parent-reported ASD symptomology. Significant group differences ( $p<.05$ after Bonferroni correction) are reported for (1) GRIN2B, (2) Pathogenic, (3) ASD Low NVIQ, and (4) ASD Average NVIQ groups. 


\begin{tabular}{|c|c|c|c|c|c|c|c|c|c|c|}
\hline & \multirow[b]{2}{*}{$\begin{array}{l}\text { (1) } \\
\text { GRIN2B }\end{array}$} & \multirow[b]{2}{*}{$\mathbf{N}$} & \multirow[b]{2}{*}{$\begin{array}{l}\text { (2) } \\
\text { Pathogenic }\end{array}$} & \multirow[b]{2}{*}{$\mathbf{N}$} & \multicolumn{3}{|c|}{ ASD No Event } & \multicolumn{3}{|c|}{ Group differences } \\
\hline & & & & & $\begin{array}{l}\text { (3) Low } \\
\text { NVIQ }\end{array}$ & $\mathbf{N}$ & $\begin{array}{l}\text { (4) } \\
\text { Average } \\
\text { NVIQ }\end{array}$ & $\mathbf{N}$ & $\mathrm{p}$ & Description \\
\hline ASD Severity (ADOS-2 CSS) & $\begin{array}{l}6.5 \\
(2.54)\end{array}$ & 12 & $6.58(2.46)$ & 96 & $\begin{array}{l}8.29 \\
(2.16)\end{array}$ & 14 & $7.32(1.85)$ & 41 & 0.450 & \\
\hline RRB & $\begin{array}{l}6.08 \\
(2.78)\end{array}$ & 12 & $6.46(2.57)$ & 96 & $\begin{array}{l}7.93 \\
(2.37)\end{array}$ & 14 & $7.7(2.15)$ & 41 & 0.928 & \\
\hline Social affect & $\begin{array}{l}7.92 \\
(1.93)\end{array}$ & 12 & $7.32(2.27)$ & 96 & $\begin{array}{l}7.93 \\
(2.59)\end{array}$ & 14 & $6.78(2.28)$ & 40 & 0.266 & \\
\hline Social skills (SRS-2) Total & $\begin{array}{l}74.88 \\
(5.92)\end{array}$ & 26 & $\begin{array}{l}78.22 \\
(11.43)\end{array}$ & 94 & $\begin{array}{l}80 \\
(10.3)\end{array}$ & 14 & $\begin{array}{l}73.07 \\
(11.67)\end{array}$ & 41 & 0.044 & $\begin{array}{l}\text { None with } \\
\text { correction (p's } \\
>.08 \text { ) }\end{array}$ \\
\hline Awareness $T$ & $\begin{array}{l}72.42 \\
(9.51)\end{array}$ & 26 & $\begin{array}{l}75.31 \\
(10.21)\end{array}$ & 94 & $\begin{array}{l}77 \\
(8.95)\end{array}$ & 14 & $\begin{array}{l}68.88 \\
(10.73)\end{array}$ & 41 & 0.006 & $4<2$ \\
\hline Cognition T & $\begin{array}{l}77.00 \\
(5.51)\end{array}$ & 26 & $\begin{array}{l}75.56 \\
(10.17)\end{array}$ & 94 & $\begin{array}{l}75.79 \\
(12.08)\end{array}$ & 14 & $\begin{array}{l}68.51 \\
(11.27)\end{array}$ & 41 & 0.002 & $4<1 \& 2$ \\
\hline Communication & $\begin{array}{l}75.04 \\
(7.06)\end{array}$ & 26 & $\begin{array}{l}78.5 \\
(11.96)\end{array}$ & 94 & $\begin{array}{l}78.71 \\
(10.79)\end{array}$ & 14 & $\begin{array}{l}72.68 \\
(12.47)\end{array}$ & 41 & 0.048 & $4<1 \& 2$ \\
\hline Motivation T & $\begin{array}{l}58.65 \\
(9.59)\end{array}$ & 26 & $\begin{array}{l}66.81 \\
(13.17)\end{array}$ & 94 & $\begin{array}{l}71.07 \\
(10.09)\end{array}$ & 14 & $\begin{array}{l}67.54 \\
(11.34)\end{array}$ & 41 & 0.004 & $1<3 \& 4$ \\
\hline Mannerisms $\mathrm{T}$ & $\begin{array}{l}75.46 \\
(9.77)\end{array}$ & 26 & $\begin{array}{l}76.83 \\
(13.85)\end{array}$ & 94 & $\begin{array}{l}79 \\
(13.08)\end{array}$ & 14 & $\begin{array}{l}73.05 \\
(13.3)\end{array}$ & 41 & 0.368 & \\
\hline ASD Screener (SCQ) & $\begin{array}{l}18.55 \\
(6.8)\end{array}$ & 20 & $\begin{array}{l}20.66 \\
(7.43)\end{array}$ & 65 & $\begin{array}{l}23.85 \\
(5.01)\end{array}$ & 13 & $\begin{array}{l}18.85 \\
(6.21)\end{array}$ & 33 & 0.035 & $\begin{array}{l}\text { None with } \\
\text { correction (p's } \\
>.07 \text { ) }\end{array}$ \\
\hline $\begin{array}{l}\text { Adaptive skills - Social } \\
\text { subdomain (VABS-2) }\end{array}$ & $\begin{array}{l}60.09 \\
(12.87)\end{array}$ & 32 & $\begin{array}{l}60.21 \\
(13.41)\end{array}$ & 96 & $\begin{array}{l}61.29 \\
(8.98)\end{array}$ & 14 & $\begin{array}{l}73.95 \\
(13.41)\end{array}$ & 41 & $<.0001$ & $1 \& 2 \& 3<4$ \\
\hline $\begin{array}{l}\text { Repetitive behaviors (RBS-R) } \\
\text { Total score }\end{array}$ & $14(9.85)$ & 15 & $\begin{array}{l}18.1 \\
(12.48)\end{array}$ & 93 & $\begin{array}{l}30.57 \\
(22.01)\end{array}$ & 14 & $\begin{array}{l}19.15 \\
(12.25)\end{array}$ & 41 & 0.008 & $1 \& 2<3$ \\
\hline Compulsive & $\begin{array}{l}2.13 \\
(2.36)\end{array}$ & 15 & 2.99 (3.42) & 93 & $\begin{array}{l}9.36 \\
(6.63)\end{array}$ & 14 & $4.78(4.3)$ & 41 & $<.0001$ & $1 \& 2<4<3$ \\
\hline Self-injurious & $\begin{array}{l}3.8 \\
(3.32)\end{array}$ & 15 & $3.16(3.57)$ & 93 & $\begin{array}{l}3.57 \\
(4.54)\end{array}$ & 14 & $2.15(2.67)$ & 41 & 0.447 & \\
\hline Sensory motor & $\begin{array}{l}5.13 \\
(3.54)\end{array}$ & 15 & $5.73(4.52)$ & 93 & $\begin{array}{l}6.36 \\
(6.69)\end{array}$ & 14 & 3.68 (2.99) & 41 & 0.193 & \\
\hline Restricted & $\begin{array}{l}0.87 \\
(1.55)\end{array}$ & 15 & $1.85(1.84)$ & 93 & $2.5(2.1)$ & 14 & $2.41(1.86)$ & 41 & 0.035 & $1<3 \& 4$ \\
\hline Sameness & $\begin{array}{l}2.07 \\
(2.12)\end{array}$ & 15 & $4.37(4.93)$ & 93 & $\begin{array}{l}8.79 \\
(8.17)\end{array}$ & 14 & $6.12(5.58)$ & 41 & 0.004 & $2 \& 1<3 ; 1<4$ \\
\hline
\end{tabular}

Table 3. ADI-R items with ASD symptomology relevant to GRIN2B. Significant chi-square results pertaining to GRIN2B are presented for ADI-R items. Significant group differences ( $p<.05$ after Bonferroni correction) are reported for (1) GRIN2B, (2) Pathogenic, (3) ASD Low NVIQ, and (4) ASD Average NVIQ groups. See SI Table 3 for complete analyses. 


\begin{tabular}{|c|c|c|c|c|c|c|c|c|c|c|}
\hline Problem & Subdomain & Item & Description & $x^{2}$ & $p$ & $\begin{array}{l}\text { (1) } \\
\text { GRIN2B }\end{array}$ & $\begin{array}{l}\text { (2) } \\
\text { Pathogenic }\end{array}$ & $\begin{array}{l}\text { (3) } \\
\text { Low } \\
\text { NVIQ }\end{array}$ & $\begin{array}{l}\text { (4) } \\
\text { Average } \\
\text { NVIQ }\end{array}$ & $\begin{array}{l}\text { Group } \\
\text { difference }\end{array}$ \\
\hline Current & $\begin{array}{l}\text { Socioemotional } \\
\text { reciprocity }\end{array}$ & 52 & $\begin{array}{l}\text { Showing and } \\
\text { directing attention }\end{array}$ & 15.47 & 0.017 & $50.0 \%$ & $37.5 \%$ & $35.7 \%$ & $9.8 \%$ & $4<1 \& 2$ \\
\hline Current & $\begin{array}{l}\text { Nonverbal } \\
\text { communication }\end{array}$ & 42 & $\begin{array}{l}\text { Pointing to express } \\
\text { interest }\end{array}$ & 16.44 & 0.012 & $62.5 \%$ & $37.5 \%$ & $35.7 \%$ & $17.1 \%$ & $4<1$ \\
\hline Current & $\begin{array}{l}\text { Routines and } \\
\text { rituals }\end{array}$ & 74 & $\begin{array}{l}\text { Difficulties with } \\
\text { minor changes in } \\
\text { routines/environment }\end{array}$ & 21.90 & 0.001 & $6.3 \%$ & $24.0 \%$ & $28.6 \%$ & $46.3 \%$ & $1 \& 2<4$ \\
\hline $\begin{array}{l}\text { Most } \\
\text { abnormal } \\
4-5 \text { years }\end{array}$ & $\begin{array}{l}\text { Socioemotional } \\
\text { reciprocity }\end{array}$ & 59 & $\begin{array}{l}\text { Appropriateness of } \\
\text { social responses }\end{array}$ & 13.91 & 0.031 & $12.5 \%$ & $50.0 \%$ & $71.4 \%$ & $50.0 \%$ & $1<2 \& 3$ \\
\hline $\begin{array}{l}\text { Most } \\
\text { abnormal } \\
4-5 \text { years }\end{array}$ & $\begin{array}{l}\text { Nonverbal } \\
\text { communication }\end{array}$ & 42 & $\begin{array}{l}\text { Pointing to express } \\
\text { interest }\end{array}$ & 19.46 & 0.003 & $81.3 \%$ & $55.4 \%$ & $71.4 \%$ & $30.0 \%$ & $\begin{array}{l}4<3<1 \\
\& 2\end{array}$ \\
\hline Lifetime & $\begin{array}{l}\text { Routines and } \\
\text { rituals }\end{array}$ & 70 & $\begin{array}{l}\text { Compulsions and } \\
\text { rituals }\end{array}$ & 17.28 & 0.008 & $6.3 \%$ & $19.8 \%$ & $50.0 \%$ & $36.6 \%$ & $1 \& 2<4$ \\
\hline Lifetime & $\begin{array}{l}\text { Routines and } \\
\text { rituals }\end{array}$ & 74 & $\begin{array}{l}\text { Difficulties with } \\
\text { minor changes in } \\
\text { routines/environment }\end{array}$ & 32.81 & $<.001$ & $12.5 \%$ & $31.3 \%$ & $64.3 \%$ & $65.9 \%$ & $\begin{array}{l}1 \& 2<4 \\
1<3\end{array}$ \\
\hline Lifetime & $\begin{array}{l}\text { Restricted } \\
\text { interests }\end{array}$ & 68 & $\begin{array}{l}\text { Circumscribed } \\
\text { interests }\end{array}$ & 18.19 & 0.006 & $31.3 \%$ & $27.1 \%$ & $42.9 \%$ & $51.2 \%$ & $1 \& 2<3$ \\
\hline Lifetime & $\begin{array}{l}\text { Sensory } \\
\text { differences }\end{array}$ & 73 & $\begin{array}{l}\text { Abnormal, } \\
\text { idiosyncratic, } \\
\text { negative response to } \\
\text { specific sensory } \\
\text { stimuli }\end{array}$ & 16.92 & 0.010 & $31.3 \%$ & $31.3 \%$ & $57.1 \%$ & $56.1 \%$ & $1<3 \& 4$ \\
\hline
\end{tabular}

Figures
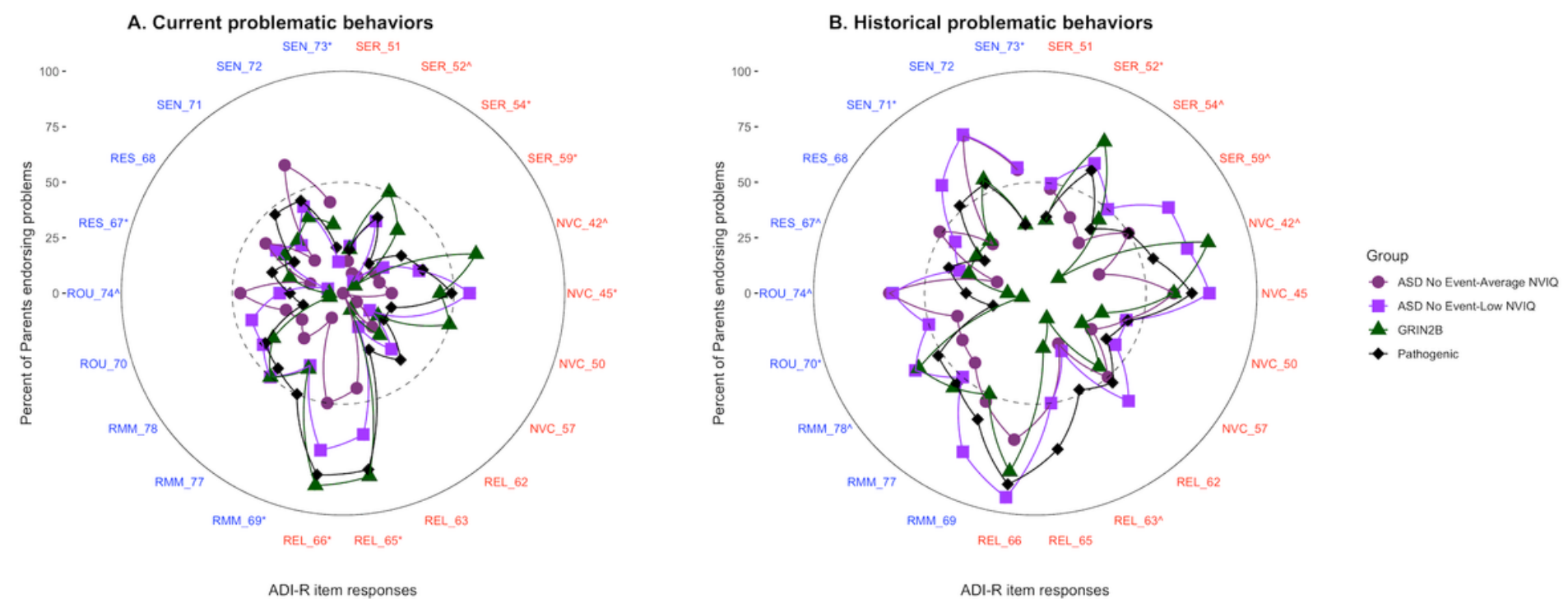

Figure 1

Parental-endorsed current (Panel A) and historical (Panel B) problems. Radar plots depict the proportion of parents endorsing problems on ADI-R items with points further from the center indicating a larger proportion and gridlines reflecting quartiles (dashed inner circle $=50 \%$ endorsed problems; solid outer circle $=100 \%$ endorsed problems). Outside the plot, item subdomain and item number are listed. Significant group differences $\left(p<.05\right.$ with Bonferroni correction) are noted with a superscript: asterisk $\left(^{*}\right)$ indicates significant results pertaining to GRIN2B and wedge $\left(^{\wedge}\right)$ indicates other effects of group. Group designations: green triangle, GRIN2B; black diamond, Pathogenic; dark purple circle, ASD No Event Average NVIQ; light purple square, ASD No Event Average NVIQ. Subdomain abbreviations: SER, socioemotional reciprocity; NVC, 
nonverbal communication; REL, relationships; RMM, repetitive motor movements; ROU, routines and rituals; RES, restricted interests; SEN, sensory differences.
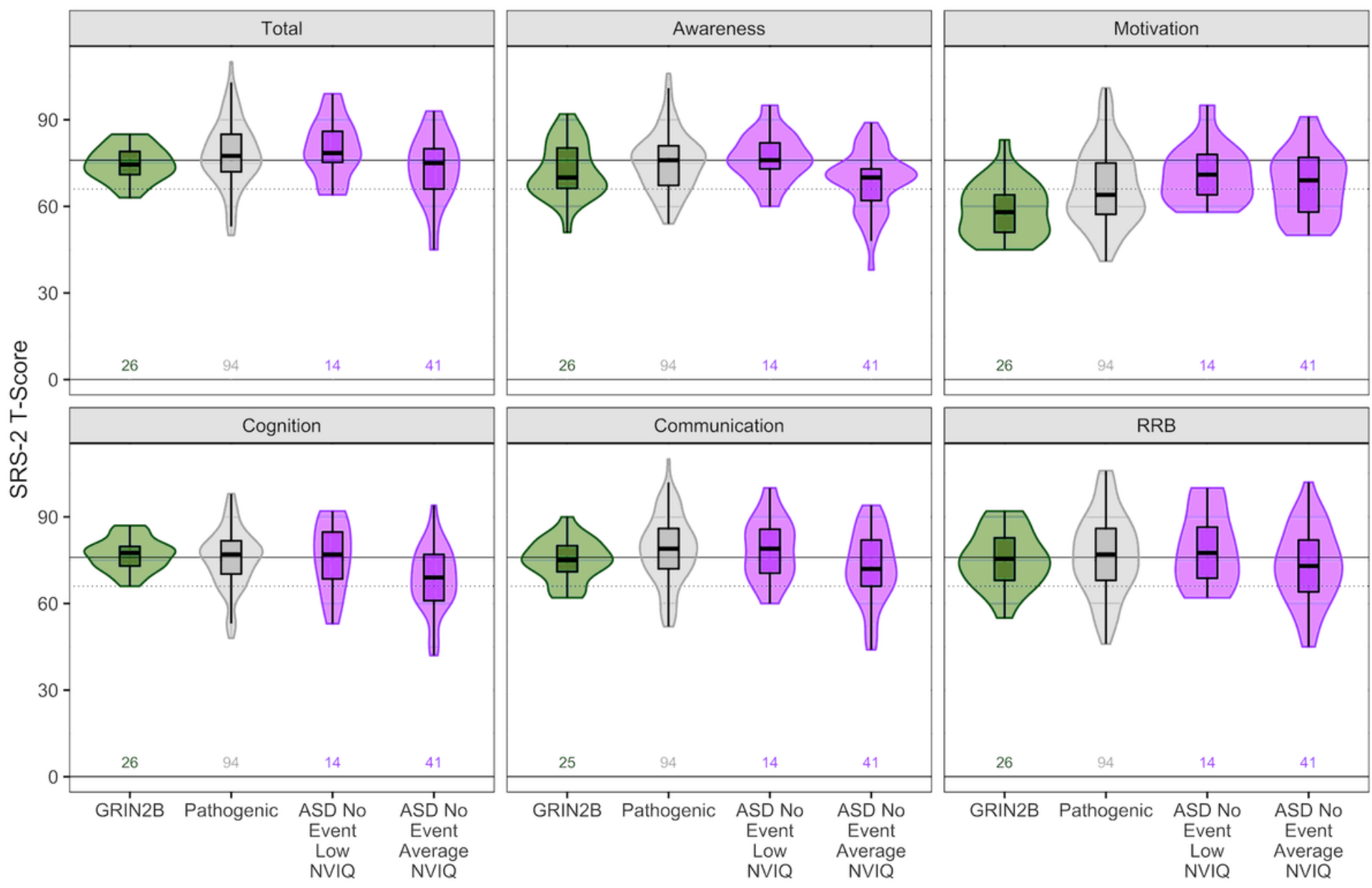

Figure 2

Parent-reported scores on social responsiveness scale (SRS-2). Group means for total and subdomain T-scores on the SRS-2 are illustrated as boxplots describing median and interquartile range. Group distribution is indicated by violin plot. For a guide, horizontal lines reference borderline range (dashed) and clinical range (solid). Number of participants contributing data are indicated at the bottom for each group.

\section{Supplementary Files}

This is a list of supplementary files associated with this preprint. Click to download.

- SITables20200906.xlsx

- SupplementaryTablesavailableinExcelfile.docx 